\title{
Functional and structural connectivity of the visual system in infants with perinatal brain injury
}

\author{
Stephanie L. Merhar ${ }^{1}$, Elveda Gozdas ${ }^{2}$, Jean A. Tkach'3 ${ }^{3}$ Karen L. Harpster ${ }^{4}$, Terry L. Schwartz ${ }^{5}$, Weihong Yuan ${ }^{2,3}$, \\ Beth M. Kline-Fath ${ }^{6}$, James L. Leach ${ }^{6}$, Mekibib Altaye ${ }^{7}$ and Scott K. Holland ${ }^{2,3}$
}

BACKGROUND: Infants with perinatal brain injury are at risk of later visual problems. Advanced neuroimaging techniques show promise to detect functional and structural alterations of the visual system. We hypothesized that infants with perinatal brain injury would have less brain activation during a visual functional magnetic resonance imaging (fMRI) task and reduced task-based functional connectivity and structural connectivity as compared with healthy controls.

METHODS: Ten infants with perinatal brain injury and 20 control infants underwent visual $\mathrm{fMRl}$ and diffusion tensor imaging (DTI) during natural sleep with no sedation. Activation maps, functional connectivity maps, and structural connectivity were analyzed and compared between the two groups.

RESULTS: Most infants in both groups had negative activation in the visual cortex during the fMRI task. Infants with brain injury showed reduced activation in the occipital cortex, weaker connectivity between visual areas and other areas of the brain during the visual task, and reduced fractional anisotropy in white matter tracts projecting to visual regions, as compared with control infants.

CONCLUSION: Infants with brain injury sustained in the perinatal period showed evidence of decreased brain activity and functional connectivity during a visual task and altered structural connectivity as compared with healthy term neonates.

\footnotetext{
I
}

nfants with brain injury sustained in the perinatal period are at risk of later visual problems, including low visual acuity, decreased binocular depth perception, abnormal eye movements, and cortical visual impairment $(1,2)$. The ability to predict visual outcome based on conventional imaging and ophthalmologic examinations in the neonatal period is poor $(1,3,4)$. Therefore, children with brain injury who could benefit from early ophthalmologic assessment and early intervention vision therapies may have delayed diagnosis and treatment of visual problems. As advanced neuroimaging techniques have become more common, the visual system has been studied in preterm and term infants. Using task-based fMRI, occipital lobe activation is reliably obtained in infants after term equivalent age $(5,6)$ but not in the preterm period $(7)$. Functional connectivity of the visual system in the neonatal period using task-based fMRI has not been previously reported. Diffusion tensor imaging (DTI) studies have shown that altered white matter integrity of the optic radiations is related to visual function in preterm infants (8), and volumetric analyses have correlated reduced occipital volumes in the neonatal period with later impaired oculomotor function (9).

Our long-term, overarching goal is to determine whether fMRI and DTI can be used as an adjunct to conventional clinical MRI to add additional information for clinicians and families about potential damage to the visual system in the neonatal period. In this study, we performed DTI and visual fMRI with a flashing light stimulus in 20 term control infants $<8$ wk postnatal age and in 10 infants with brain injury (both term and preterm) at $<48 \mathrm{wk}$ postmenstrual age. Our hypothesis was that we would find differences in activation, functional connectivity, and structural connectivity between the two groups, with less brain activation and weaker functional and structural connectivity in infants with brain injury vs. healthy controls. We formulated this hypothesis based on the theory that the infants with brain injury would have lost neurons and the clusters of activation would be smaller, leading to less vascular response and thus reduced amplitude BOLD signal responses.

\section{RESULTS}

\section{Participants}

The control group consisted of 20 healthy term infants scanned at $<48 \mathrm{wk}$ postmenstrual age with a mean gestational age of $39 \mathrm{wk}$ and a mean postmenstrual age of $44.7 \mathrm{wk}$ at the time of scan. $60 \%$ were female and $40 \%$ were male. The 10 infants with brain injury consisted of 6 term infants and 4 preterm infants all scanned at $<48 \mathrm{wk}$ postmenstrual age, with a mean postmenstrual age of $44.2 \mathrm{wk}$ at the time of scan. $60 \%$ were female

\footnotetext{
'Perinatal Institute, Division of Neonatology, Cincinnati Children's Hospital Medical Center, Cincinnati, Ohio; ${ }^{2}$ Pediatric Neuroimaging Research Consortium, Cincinnati Children's Hospital Medical Center, Cincinnati, Ohio; ${ }^{3}$ Imaging Research Center, Cincinnati Children's Hospital Medical Center, Cincinnati, Ohio; ${ }^{4}$ Division of Occupational and Physical Therapy, Cincinnati Children's Hospital Medical Center, Cincinnati, Ohio; ${ }^{5}$ Division of Ophthalmology, Cincinnati Children's Hospital Medical Center, Cincinnati, Ohio; ${ }^{6}$ Department of Radiology and Medical imaging, Cincinnati Children's Hospital Medical Center, Cincinnati, Ohio; ${ }^{7}$ Division of Biostatistics and Epidemiology, Cincinnati Children's Hospital Medical Center, Cincinnati, Ohio. Correspondence: Stephanie L. Merhar (stephanie.merhar@cchmc.org)

Received 10 August 2015; accepted 23 December 2015; advance online publication 6 April 2016. doi:10.1038/pr.2016.49
} 


\section{Articles | Merhar et al.}

and $40 \%$ were male. Demographic and MRI characteristics of the brain injury group are described in Table 1.

\section{Visual Task fMRI}

On individual analysis, 14/20 control participants and 8/10 of those with brain injury had negative brain activation in the expected visual cortical areas. One control participant had positive activation in the visual cortex, and the rest had no activation. Figure 1 shows the group average activation maps for the controls (a), infants with brain injury (b), and the difference between the groups (c), as measured by fMRI during visual stimulation, using a mixed effects model. The controls had a significantly higher percent signal change, which represents the change in BOLD signal intensity from baseline, of $1.78 \%$ as compared with $1.4 \%$ for the brain injury group, $P=0.007$.

\section{Functional Connectivity}

The control group had stronger connectivity between the visual areas and other areas of the brain, as shown in the task-based functional connectivity maps in Figure 2. Functional connectivity was derived from BOLD time courses modeled during the task in the block design using a general linear model. We examined several areas of the brain known to be related to vision for their functional connectivity with other brain regions in the network, indicated by the green circles in each panel of Figure 2. In the control group, the lingual gyrus (a), supramarginal gyrus (b), cuneus (c), superior occipital gyrus (d), middle occipital gyrus (e), and inferior occipital gyrus (f) were found to have strong functional connectivity with other regions, as shown in Figure 2 (left). By contrast, these regions in children with brain injury showed very few strong connections with other brain regions, shown in Figure 2 (right).

\section{Structural Connectivity}

Using DTI, we computed the fractional anisotropy (FA) of water diffusion in each subject. Figure 3 shows selected images highlighting the difference in FA along the skeleton of peak FA values averaged over all participants (shown in green) using tract-based spatial statistics. The control group demonstrated higher FA (highlighted in red) in the genu of the corpus callosum and the left optic radiation. Using region of interest (ROI)-based analysis, we showed that the brain injury group had lower FA in the optic radiations than the control group. The mean FA in the brain injury group was $0.21 \pm 0.09$, vs. $0.30 \pm 0.04$ in the controls, $P=0.0004$.

\section{DISCUSSION}

Normal vision requires intact anterior (eyes, optic nerves, optic chiasm) and posterior (lateral geniculate nucleus, optic radiations, and primary visual cortex) pathways. Visual information is then relayed to association cortices or to the extrageniculate visual system (2). The corpus callosum allows for integration of the two halves of the visual field (10). In cortical

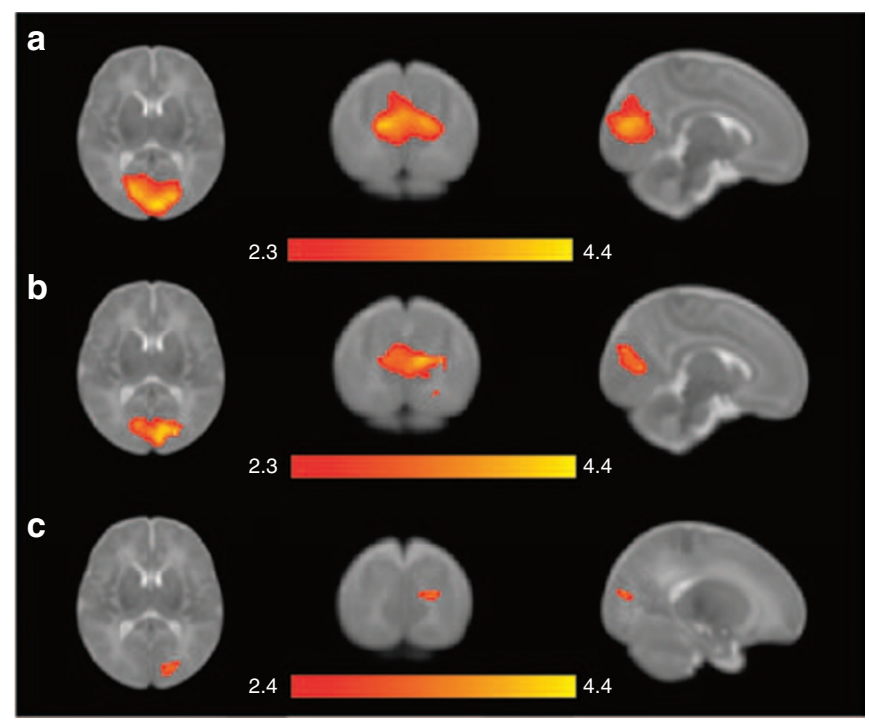

Figure 1. Z-score maps of brain activation during the visual fMRI task. The top panel (a) shows controls, the middle panel (b) shows infants with brain injury, and the bottom panel (c) shows the difference between the two. Red areas represent regions of activation during the visual task. $Z$ maps are thresholded using clusters determined by $Z>2.4$ and a false-discovery rate corrected significance threshold of $P=0.05$.

Table 1. Characteristics of brain injury group

\begin{tabular}{|c|c|c|c|c|c|c|}
\hline Patient & Gender & $\begin{array}{l}\text { GA at birth } \\
\text { (weeks) }\end{array}$ & $\begin{array}{l}\text { Corrected age } \\
\text { at scan (weeks) }\end{array}$ & Type of brain injury & $\begin{array}{c}\text { Visual areas } \\
\text { affected on MRI? }\end{array}$ & $\begin{array}{l}\text { Severity of damage } \\
\text { to visual areas }\end{array}$ \\
\hline 1 & $\mathrm{~F}$ & 40.4 & 46.9 & Hypoxic ischemic encephalopathy & Left & Mild \\
\hline 3 & $\mathrm{~F}$ & 29.6 & 39.7 & Periventricular leukomalacia & Bilateral & Moderate \\
\hline 4 & M & 29.7 & 41.9 & Right grade $4 \mathrm{IVH}$, mild PVL & None & Normal \\
\hline 7 & $\mathrm{~F}$ & 27 & 40.1 & Left grade 4 IVH & None & Normal \\
\hline 8 & M & 39 & 45.4 & Diffuse cerebral white matter signal abnormality & Bilateral & Moderate \\
\hline 9 & $\mathrm{~F}$ & 37.7 & 48 & Diffuse encephalomalacia due to herpes & Bilateral & Severe \\
\hline 10 & M & 37.9 & 45.4 & Hypoxic ischemic encephalopathy & Bilateral & Severe \\
\hline
\end{tabular}

GA, gestational age; IVH, intraventricular hemorrhage; MRI, magnetic resonance imaging; PCA, posterior cerebral artery; PVL, periventricular leukomalacia. 
a

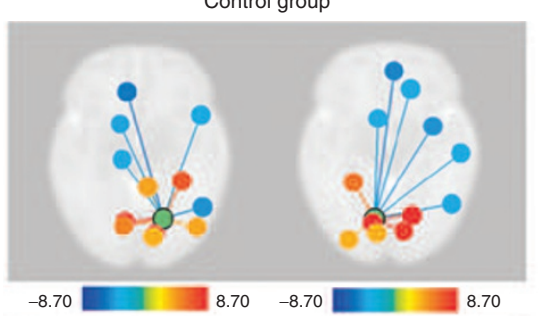

b

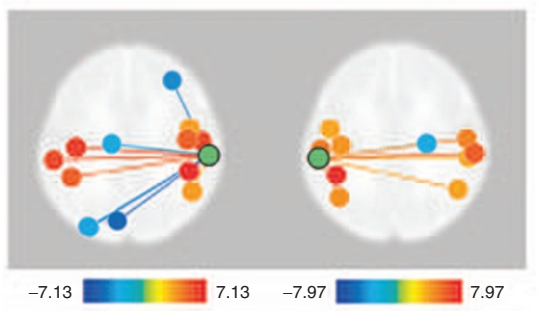

C

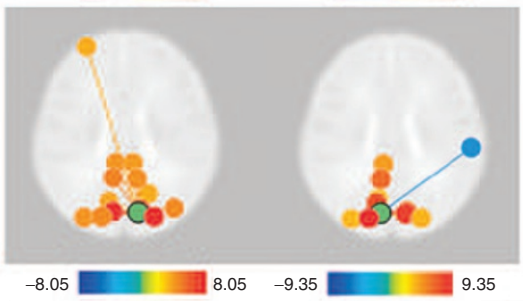

d

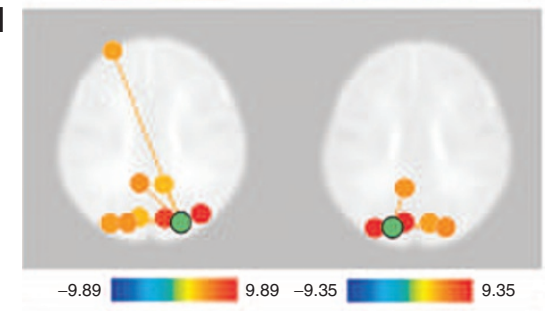

e

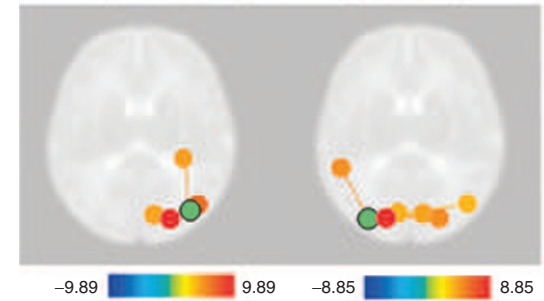

f

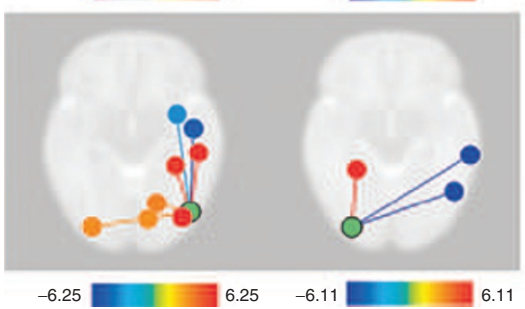

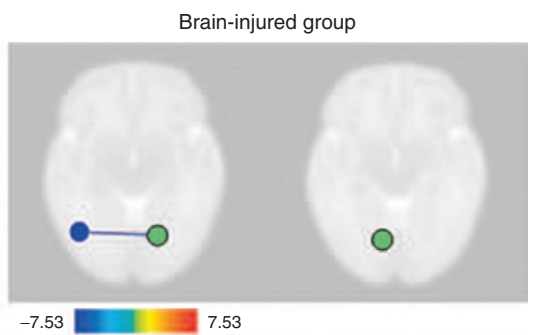
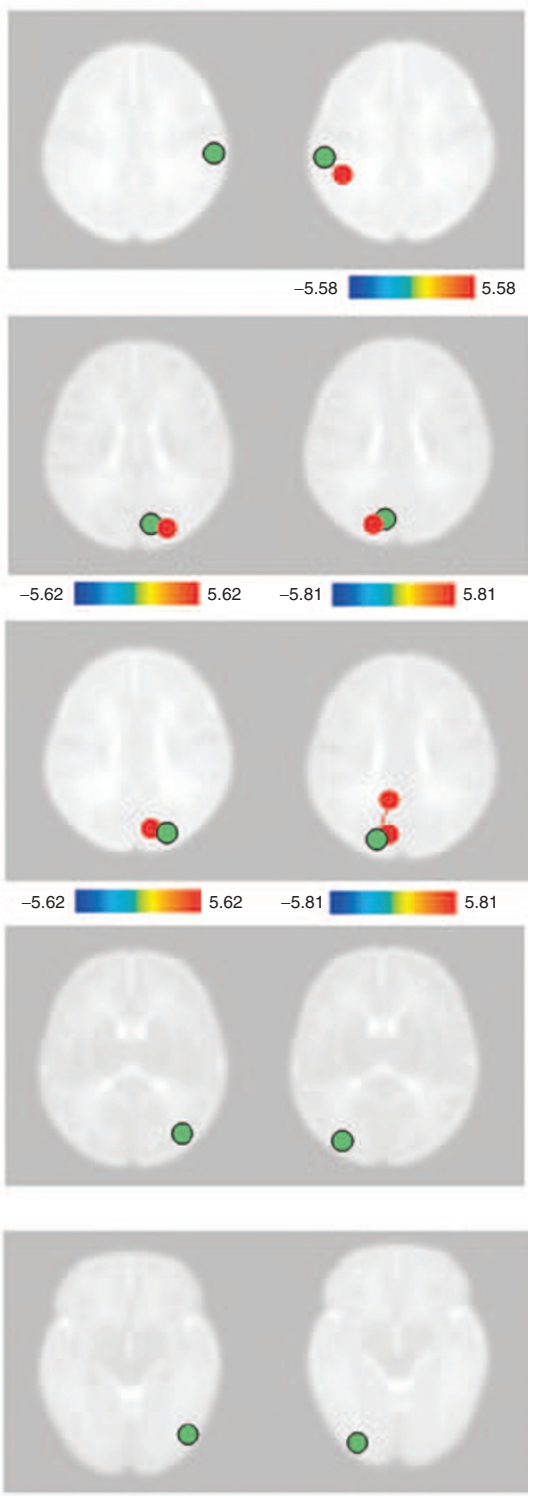

Figure 2. Functional connectivity maps derived from BOLD time courses during visual task. Colored circles represent nodes showing connectivity. Seeds are indicated by the green circles for each panel: $\mathbf{a}=$ lingual gyrus, $\mathbf{b}=$ supramarginal gyrus, $\mathbf{c}=$ cuneus, $\mathbf{d}=$ superior occipital gyrus, $\mathbf{e}=$ middle occipital gyrus, $\mathbf{f}=$ inferior occipital gyrus. Colors represent the strength of connectivity measured as a partial correlation coefficient of the time course in the connected regions (warm colors = positive connectivity, cool colors = negative connectivity). The range of correlations represented on the color scale is determined by a significance threshold of $P<0.05$ (corrected for multiple comparisons using false-discovery rate). Nodes that are connected by lines in the figure represent connections between the seed and regions in a different plane.

visual impairment, visual loss is due to injury to the primary visual cortex (V1) and/or extrastriate visual cortical areas (V2, V3, V4, V5). In the term infant, cortical visual impairment is most commonly due to hypoxic-ischemic injury or severe hypoglycemia $(11,12)$. In the preterm neonate, cortical visual impairment is often due to brain injury directly impacting the visual pathways, most often the optic radiations or interruption of the thalamocortical fibers due to intraventricular and/or intraparenchymal hemorrhage (grade 3-4 IVH) $(2,12)$. In our study, we compared fMRI and DTI in healthy 


\section{Articles | Merhare tal.}

term-born control infants with term and preterm infants with brain injury. Other investigators have attempted to use task based fMRI to evaluate the visual system in preterm and term neonates with varying degrees of success. In preterm infants before term equivalent age, activation of the visual cortex in response to a strong visual stimulus is inconsistent $(7,13)$. From term age up until several months or years of life, BOLD response can often be elicited but most authors have found a positive BOLD signal in these infants (5-7,13-15). In contrast, we found fairly consistent negative visual BOLD activation in the medial occipital lobe in our population. Negative BOLD signal during activation can be caused by a decrease in cerebral blood flow, increase in cerebral blood volume, or increase in oxygen extraction. Recent animal studies demonstrate that, unlike in adults, the delivery of oxygenated hemoglobin does not overcompensate for the elevated local metabolic demand in the newborn (16). An increase in the local deoxyhemoglobin concentration results from focal brain stimulation, leading to a negative BOLD response, as was observed in our study. Another possible explanation is that the strong visual stimulus creates a very high local metabolic demand, and blood flow is unable to increase sufficiently in the neonates to compensate. In addition, recent data shows that the coupling between the BOLD signal and hemodynamic response is age-dependent in children, being weaker in younger children, which could imply that this response is not sufficiently mature in neonates to meet metabolic demand (17). Notably, in one study, sleeping adults showed a negative BOLD response to the same strong visual stimulus we used, which may be due to direct neural inhibition of the visual cortex during sleep (18).

In a heterogeneous group of infants and older children with suspected visual deficit, visual impairment was variably associated with smaller cortical volumes of activation on fMRI (13). In former preterm infants with PVL, visual impairment evaluated near the time of fMRI correlated with abnormal BOLD response (19). Both of these studies are consistent with our results as shown in Figure 1, in which the control group had less activation during the visual task than the brain injury group. However, these studies were both done in infants/children with established visual deficits.

Neural networks can be characterized by observing temporal fluctuations in the functional MRI signal to identify areas that are functionally connected. Correlation found between two regions suggests that the regions work together and have similar temporal responses. Negative connectivity refers to temporally inverse correlation in BOLD fluctuation, where when one node demonstrates increased BOLD signal intensity relative to baseline, the other node shows lower BOLD signal intensity relative to baseline. Functional connectivity can be computed for resting state BOLD time courses, as well as time courses derived from the task in the block design, as we did here. In adults, networks have been identified in regions of the brain involved in vision, hearing, sensation, motor function, attention, and behavior (20). Resting state networks that correspond with those seen in adults are known to be present at term $(21,22)$ and even prior to birth (23). A resting state network located in the medial aspects of the occipital cortex appears as early as $24-26$ wk gestation $(22,23)$ and is similar to the visual network found in the adult brain (20). Intact networks are thought to be necessary for normal neural processing, and disrupted networks have been seen in preterm infants as compared with normal controls (22). We evaluated the connectivity of the visual system using task-based fMRI and found that healthy term neonates have a more robust visual network than infants with perinatal brain injury. We expect that this finding will have implications for visual outcomes in the infants with brain injury, though we will not have outcome measures from this group for another $2-3 \mathrm{y}$.

Diffusion tensor imaging provides information about white matter architecture and integrity. A measure derived from DTI, FA, reflects the degree to which the motion of water is directionally restricted. Higher FA values are typically interpreted to indicate a more organized and intact fiber structure. However, higher FA values may also reflect the presence of fewer crossing fibers within the voxel (24). Using tract-based spatial statistics, we found that FA values in the infants with brain injury were significantly lower than FA values in the control group in several white matter regions relevant for vision, including the corpus callosum and the left optic radiation. Several studies have shown that FA in the optic radiations correlates with visual fixation and visual assessment scores at the time of scan in preterm infants $(4,8,25)$, and one of these studies found that the relationship appears specific to the optic radiation, as FA was not related to visual function in any other region (8). In former preterm infants studied in adolescence, lower FA in the optic radiations was associated with increased odds of impaired vision. Volume, surface area, and thickness of the primary visual cortex were altered, but not associated with impaired vision in this population (26).

The main limitations of our study include small sample size, heterogeneous etiology of brain injury, and lack of longitudinal outcome data to determine long-term visual function. Due to small sample size and variability of pathology, visualization of results in the figures is highly threshold dependent. We used a strict threshold in order to avoid misrepresenting sources of activation. Despite the considerable heterogeneity of the groups, we still found a significant difference in patterns of activation, suggesting that the effect is strong despite the considerable variance. To address the limitations of our study, we plan on conducting further studies with a larger sample size and a more homogenous population using a prospective, longitudinal design. We have shown on a group basis that fMRI and DTI can discriminate between controls and infants with brain injury, but in order to individualize prediction (for example, using machine learning), a larger sample size would be necessary. It is possible that the flash stimulus we used may be biased to identify anterior visual pathway disease (such as optic nerve and retinal damage) and future directions could include more complex visual stimuli to obtain maximal response from $\mathrm{V} 1$ and association areas.

In conclusion, we have shown that a visual paradigm can reliably cause negative activation in the visual cortex in sleeping 


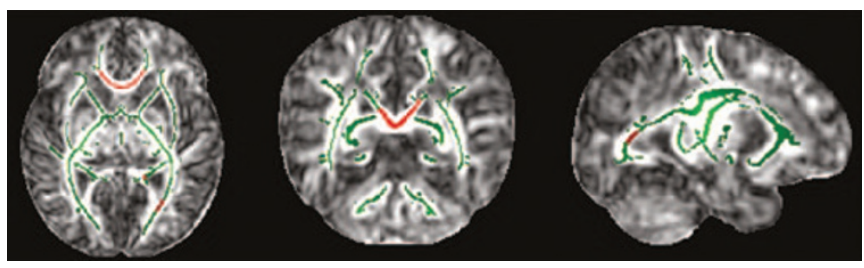

Figure 3. Tract-based spatial statistics map showing the diffusion tensor imaging skeleton based on peak fractional anisotropy value for all participants in green and difference in fractional anisotropy between the control group and the brain injury group in red. Areas in red represent the areas where the control group had higher fractional anisotropy than the brain injury group (genu of the corpus callosum, left internal capsule and left optic radiation), with a threshold of $P<0.05$ corrected for multiple comparisons using family-wise error rate.

unsedated neonates scanned at $<48$ wk postmenstrual age, and that infants with brain injury sustained in the perinatal period have altered task-based functional and structural connectivity as compared with healthy term neonates.

\section{METHODS}

\section{Participants}

Twenty term control infants and 10 infants with brain injury were scanned using fMRI with a visual stimulation paradigm before $48 \mathrm{wk}$ postmenstrual age. The protocol was approved by the Cincinnati Children's Hospital Institutional Review Board and informed parental consent was obtained. All imaging was undertaken during unsedated natural sleep (27). Hearing protection was provided with earplugs and headphones.

\section{MRI Acquisition Parameters}

MRI data were all acquired on a 3 T Philips Achieva MRI scanner (Phillips Medical Systems, Best, the Netherlands) in the Imaging Research Center at Cincinnati Children's Hospital using a 32 channel phased array head coil. Infants were swaddled for scanning and placed in the head coil with ear phones and ear muffs for hearing protection. A high spatial resolution (1 mm isotropic) 3D T1-weighted scan was obtained using an inversion recovery gradient echo imaging method for anatomic reference.

\section{fMRI}

fMRI was performed in the axial plane using a single shot gradient echo echo-planar imaging sequence with echo time $35 \mathrm{~ms}$, repetition time $2 \mathrm{~s}$, slice thickness $3 \mathrm{~mm}$, flip angle $=90^{\circ}$, field of view $180 \times 180 \mathrm{~mm}$, matrix size $64 \times 64$, voxel size $2.8 \times 2.8 \times 3 \mathrm{~mm}, 36$ slices, SENSE Factor $=2$; receiver $\mathrm{BW}=3423.6 \mathrm{~Hz} /$ pixel.

\section{Visual Task Paradigm}

The visual paradigm was administered in a periodic block design consisting of 5.5 cycles of $30 \mathrm{~s}$ ON/OFF conditions. For the ON condition, a visual stimulus ( $8 \mathrm{~Hz}$ flashing lights) was delivered via a LCD display blinking white, through closed eyelids, contrasted with total darkness during the OFF condition. The total scan time was $5 \mathrm{~min}$ $30 \mathrm{~s}$, during which 165 BOLD image volumes were obtained.

\section{DTI}

DTI was obtained in the axial plane using a b value of $800 \mathrm{~s} / \mathrm{mm}^{2}$ and 61 directions uniformly distributed over a sphere using an electrostatic repulsive model, interleaved with $7 \mathrm{~b}=0$ scans. Scan parameters: $\mathrm{TR} / \mathrm{TE}=6389 \mathrm{~ms} / 77 \mathrm{~ms}$, FOV $224 \times 224 \mathrm{~mm}$, matrix size $112 \times 109$, $60-2 \mathrm{~mm}$ slices, voxel size $1.75 \times 1.75 \times 2.0 \mathrm{~mm}$, SENSE factor $=3$; receiver $\mathrm{BW}=1752.6 \mathrm{~Hz} /$ pixel.

\section{Analysis}

fMRI data were analyzed using FEAT (fMRI Expert Analysis Tool, Version 5.0.8) which was part of the FSL processing package (FMRIB, Oxford, UK) (28). Standard preprocessing steps were applied to all data steps as implemented in FEAT: motion correction, nonbrain tissue removal, spatial smoothing, normalization (grand mean scaling of the timeseries), and high pass filtering. Data sets were assessed and corrected for motion using MCFLIRT (FSL's intramodal motion correction tool), which estimates absolute and relative displacement through the entire image time-series. After motion correction, skull stripping was performed using the BET function implemented in FSL. Images were temporally high-pass filtered and smoothed using a $6 \mathrm{~mm}$ Gaussian kernel full-width at half maximum algorithm. We used FLIRT (FMRIB's Linear Image Registration Tool) to register the functional data to the neonate atlas (29) with 12 degrees of freedom affine transformation. For each subject, data were fitted to the general linear model as the first level analysis with the six motion parameters modeled as covariates. The second level analysis was performed based on mixed effect analysis using FLAME. For each subject, first level activation maps were merged in a higher-level mixed effect analysis using FLAME (FMRI's local analysis of mixed effects) to evaluate group differences. All subjects were included in the group analysis, but only negative BOLD responses were included in the first-level analysis. To ensure that the mixed effect analysis was valid given the heterogeneity of our population, we used the Randomise routine within the FSL program to perform a nonparametric permutation-based statistical analysis, with no difference in the results.

For the visual functional connectivity analysis, all image processing was performed using SPM8 (Welcome Department of Imaging Neuroscience, London, UK). The functional image series were corrected for head motion by realigning all images to the mean of all functional volumes. The mean functional image was coregistered to a corresponding T1-weighted high-resolution image. T1-weighted images were segmented using neonatal tissue probability maps (29). Finally, the images were normalized to the neonatal template, resliced to a voxel size $2 \times 2 \times 2 \mathrm{~mm}$, and smoothed with a full width at half maximum Gaussian Smoothing kernel of $6 \mathrm{~mm}$. After pre-processing for each subject, a general linear model was used to regress the time course of visual task vs. rest as the main effect and the realignment parameters as regressors of no interest. SPM.mat files of each subject were imported into CONN (30) to process ROI-to-ROI connectivity analyses. This toolbox implements a noise reduction method (aCompCor) (31) that accounts for physiological noise, and the covariates were included with a principal components analyses reduction of the signal from subject specific white matter and CSF masks. The residual BOLD timeseries was then band-pass filtered $(0.008<\mathrm{f}<\mathrm{inf})$. Additionally, six motion parameter timeseries were condensed into a single vector and entered into second level analysis to reduce further motion effects. ROI based analysis was performed by grouping voxels into 90 ROIs based upon a neonate.aal (Automated Anatomical Labeling) atlas. Bi-variate correlations were calculated between each pair of ROIs. All ROIs were imported as possible connections for our selected ROIs. The unpaired $t$-test was used with a threshold set at $P<0.05$ false discovery rate corrected to determine whether significant differences existed between the control and brain injured groups.

For the DTI analysis, images were preprocessed in the FSL software (FMRIB Software Library, FMRI, Oxford, UK) including correction for eddy current and head motion (32) and skull stripping (33). Preprocessed images were subjected to tensor decomposition for generating FA maps in FDT (34) toolbox (FMRIB's Diffusion Toolbox). Tract-based spatial statistics (35) was then used to test group differences of DTI measures. All subjects' FA maps were aligned into the JHU_ neonate_nonlinear_fa template (36) using a nonlinear registration in FNIRT (FMRIB's Non_linear Registration Tool) (37) and then the affine transform to MNI space was applied. After transformation into MNI space, a mean FA image was created and thinned to generate a mean FA skeleton of white matter tracts. Voxel-wise statistical analysis across subjects was carried out for voxels with FA $>0.2$ to include only major fiber bundles. Nonparametric permutation based statistical analysis was performed with Randomise (38), and statistical results are reported as corrected $P$ values at $P<0.05$ after controlling for family-wise error rate.

\section{STATEMENT OF FINANCIAL SUPPORT}

Financial support for this project was received from the Thrasher Research Fund, Salt Lake City, UT, project 9190.

Disclosure: The authors have no disclosures or conflicts of interest. 


\section{Articles | Merhare al}

\section{REFERENCES}

1. Mercuri E, Atkinson J, Braddick O, et al. Visual function in full-term infants with hypoxic-ischaemic encephalopathy. Neuropediatrics 1997;28:155-61.

2. Chau V, Taylor MJ, Miller SP. Visual function in preterm infants: visualizing the brain to improve prognosis. Doc Ophthalmol 2013;127:41-55.

3. Mercuri E, Atkinson J, Braddick O, et al. Basal ganglia damage and impaired visual function in the newborn infant. Arch Dis Child Fetal Neonatal Ed 1997;77:F111-4.

4. Bassi L, Ricci D, Volzone A, et al. Probabilistic diffusion tractography of the optic radiations and visual function in preterm infants at term equivalent age. Brain 2008;131(Pt 2):573-82.

5. Martin E, Joeri P, Loenneker T, et al. Visual processing in infants and children studied using functional MRI. Pediatr Res 1999;46:135-40.

6. Yamada H, Sadato N, Konishi Y, et al. A milestone for normal development of the infantile brain detected by functional MRI. Neurology 2000;55:218-23.

7. Lee W, Donner EJ, Nossin-Manor R, Whyte HE, Sled JG, Taylor MJ. Visual functional magnetic resonance imaging of preterm infants. Dev Med Child Neurol 2012;54:724-9.

8. Groppo M, Ricci D, Bassi L, et al. Development of the optic radiations and visual function after premature birth. Cortex 2014;56:30-7.

9. Shah DK, Guinane C, August P, et al. Reduced occipital regional volumes at term predict impaired visual function in early childhood in very low birth weight infants. Invest Ophthalmol Vis Sci 2006;47:3366-73.

10. Pietrasanta M, Restani L, Caleo M. The corpus callosum and the visual cortex: plasticity is a game for two. Neural Plast 2012;2012:838672.

11. Tam EW, Widjaja E, Blaser SI, Macgregor DL, Satodia P, Moore AM. Occipital lobe injury and cortical visual outcomes after neonatal hypoglycemia. Pediatrics 2008;122:507-12.

12. Lehman SS. Cortical visual impairment in children: identification, evaluation and diagnosis. Curr Opin Ophthalmol 2012;23:384-7.

13. Born AP, Miranda MJ, Rostrup E, et al. Functional magnetic resonance imaging of the normal and abnormal visual system in early life. Neuropediatrics 2000;31:24-32.

14. Born P, Leth H, Miranda MJ, et al. Visual activation in infants and young children studied by functional magnetic resonance imaging. Pediatr Res 1998;44:578-83.

15. Erberich SG, Friedlich P, Seri I, Nelson MD Jr, Blüml S. Functional MRI in neonates using neonatal head coil and MR compatible incubator. Neuroimage 2003;20:683-92.

16. Kozberg MG, Chen BR, DeLeo SE, Bouchard MB, Hillman EM. Resolving the transition from negative to positive blood oxygen level-dependent responses in the developing brain. Proc Natl Acad Sci USA 2013;110:4380-5.

17. Schmithorst VJ, Vannest J, Lee G, et al.; CMIND Authorship Consortium. Evidence that neurovascular coupling underlying the BOLD effect increases with age during childhood. Hum Brain Mapp 2015;36:1-15.

18. Born AP, Law I, Lund TE, et al. Cortical deactivation induced by visual stimulation in human slow-wave sleep. Neuroimage 2002;17:1325-35.

19. Yu B, Guo Q, Fan G, Liu N. Assessment of cortical visual impairment in infants with periventricular leukomalacia: a pilot event-related FMRI study. Korean J Radiol 2011;12:463-72.
20. Damoiseaux JS, Rombouts SA, Barkhof F, et al. Consistent resting-state networks across healthy subjects. Proc Natl Acad Sci USA 2006;103: 13848-53.

21. Fransson P, Skiöld B, Horsch S, et al. Resting-state networks in the infant brain. Proc Natl Acad Sci USA 2007;104:15531-6.

22. Smyser CD, Inder TE, Shimony JS, et al. Longitudinal analysis of neural network development in preterm infants. Cereb Cortex 2010;20:2852-62.

23. Thomason ME, Grove LE, Lozon TA Jr, et al. Age-related increases in long-range connectivity in fetal functional neural connectivity networks in utero. Dev Cogn Neurosci 2015;11:96-104.

24. Dudink J, Kerr JL, Paterson K, Counsell SJ. Connecting the developing preterm brain. Early Hum Dev 2008;84:777-82

25. Berman JI, Glass HC, Miller SP, et al. Quantitative fiber tracking analysis of the optic radiation correlated with visual performance in premature newborns. AJNR Am J Neuroradiol 2009;30:120-4.

26. Kelly CE, Cheong JL, Molloy C, et al.; Victorian Infant Collaborative Study Group. Neural correlates of impaired vision in adolescents born extremely preterm and/or extremely low birthweight. PLoS One 2014;9:e93188.

27. Vannest J, Rajagopal A, Cicchino ND, et al.; CMIND Authorship Consortium. Factors determining success of awake and asleep magnetic resonance imaging scans in nonsedated children. Neuropediatrics 2014;45:370-7.

28. Smith SM, Jenkinson M, Woolrich MW, et al. Advances in functional and structural MR image analysis and implementation as FSL. Neuroimage 2004;23 Suppl 1:S208-19.

29. Shi F, Yap PT, Wu G, et al. Infant brain atlases from neonates to 1- and 2-year-olds. PLoS One 2011;6:e18746.

30. Whitfield-Gabrieli S, Nieto-Castanon A. Conn: a functional connectivity toolbox for correlated and anticorrelated brain networks. Brain Connect 2012;2:125-41.

31. Behzadi Y, Restom K, Liau J, Liu TT. A component based noise correction method (CompCor) for BOLD and perfusion based fMRI. Neuroimage 2007;37:90-101.

32. Jenkinson $M$, Smith S. A global optimisation method for robust affine registration of brain images. Med Image Anal 2001;5:143-56.

33. Smith SM. Fast robust automated brain extraction. Hum Brain Mapp 2002;17:143-55

34. Behrens TE, Woolrich MW, Jenkinson M, et al. Characterization and propagation of uncertainty in diffusion-weighted MR imaging. Magn Reson Med 2003;50:1077-88.

35. Smith SM, Jenkinson M, Johansen-Berg H, et al. Tract-based spatial statistics: voxelwise analysis of multi-subject diffusion data. Neuroimage 2006;31:1487-505.

36. Oishi K, Mori S, Donohue PK, et al. Multi-contrast human neonatal brain atlas: application to normal neonate development analysis. Neuroimage 2011;56:8-20.

37. Rueckert D, Sonoda LI, Hayes C, Hill DL, Leach MO, Hawkes DJ. Nonrigid registration using free-form deformations: application to breast $\mathrm{MR}$ images. IEEE Trans Med Imaging 1999;18:712-21.

38. Nichols TE, Holmes AP. Nonparametric permutation tests for functional neuroimaging: a primer with examples. Hum Brain Mapp 2002;15:1-25. 